\title{
Interleukin 18 maintains a long-standing inflammation in coeliac disease patients
}

A. J. León,$^{\star}$ J. A. Garrote, ${ }^{* \dagger}$

A. Blanco-Quirós, ${ }^{\star}$ C. Calvo, ${ }^{\star \star}$

L. Fernández-Salazar, ${ }^{\S}$ A. Del Villar,

A. Barrera ${ }^{\star *}$ and E. Arranz

${ }^{*}$ Department of Paediatrics and Immunology,

and Institute of Biology and Molecular

Genetics (IBGM), Universidad de Valladolid,

Valladolid, Spain, ${ }^{\dagger}$ Research Unit, ${ }^{\ddagger}$ Paediatric

Gastroenterology Clinics, and ${ }^{\S}$ Digestive Diseases

Unit, Hospital Clínico Universitario, Valladolid,

Spain, and 'Pathology, and ${ }^{* *}$ General and

Digestive Surgery Units, Hospital Universitario

Rio-Hortega, Valladolid, Spain

Accepted for publication 28 September 2006

Correspondence: E Arranz, Department of Pae-

diatrics and Immunology-IBGM, University of

Valladolid, C/Ramón y Cajal 7, 47005-

Valladolid, Spain.

E-mail: earranz@med.uva.es

\begin{abstract}
Summary
Dietary gluten induces an early response in the intestine of coeliac disease patients $(\mathrm{CD})$, within a few hours, and this is driven by high levels of proinflammatory cytokines, including IFN $\gamma$ and IL-15, as has been thoroughly shown by gluten stimulation of biopsy explants. Our aim was to identify the immune mediators involved in the long-standing inflammation in untreated CD patients at diagnosis. mRNA and protein levels of TNF $\alpha$, IL-12(p35), IL-12(p40), IL-15, IL-18 and IL-23(p19) were quantified in biopsies from active CD patients, $C D$ patients on a gluten-free diet (GFD), healthy controls, and patients with non-CD inflammation and mild histological changes in the intestine. Biopsies from $\mathrm{CD}$ patients on a GFD were also stimulated in vitro with gliadin, and protein expression of IL-15 and IL-18 was analysed. Levels of IL-12 and IL-23 mRNA are nearly absent, and TNF $\alpha$ levels remain unchanged among different groups. Both the active and inactive forms of IL-18 protein have been found in all samples from active $\mathrm{CD}$, and protein expression was only localized within the crypts. Levels of IL-15 mRNA remain unchanged, and protein expression, localized within the lamina propria, is found in a small number of samples. In vitro stimulation with gluten induces the expression of IL-15 and IL-18. In active CD, the early response following gluten intake characterized by high IFN $\gamma$ levels is driven by IL-18, and probably IL-15, and this alternates with periods of long-standing inflammation with moderate IFN $\gamma$ levels, maintained by IL-18 alone.
\end{abstract}

Keywords: coeliac disease, cytokines, intestinal mucosa, messenger RNA

\section{Introduction}

The mucosal damage in coeliac disease (CD) is caused by an inappropriate immune reaction to dietary gluten $[1,2]$, leading to the extravasation of immune cells into the affected areas of the mucosa, and tissue remodelling [3]. Intestinal inflammation in $\mathrm{CD}$ may consist of alternating peaks of immune activity (both innate and adaptative) linked to gluten ingestion, followed by long-standing inflammation during periods of fasting. Early responses have been studied in experimental models by using gluten challenge of biopsy explants, and the implication of cytokines such as TNF $\alpha$ and IL-15 has been stated [4,5]. Long-standing inflammation has been studied in biopsies from untreated $\mathrm{CD}$ patients at diagnosis, and raised levels of IFN $\gamma$, above those of healthy controls, have been reported [6,7], although less information is available regarding cytokine regulation in this phase.

We have investigated the expression levels of several inflammatory cytokines, which are known to be involved in
IFN $\gamma$ production and mucosal inflammation, including TNFo, IL-12, IL-15, IL-18 and IL-23. Previous reports have studied the role of TNF $\alpha[5,8]$, IL-15 $[9,10]$ and IL-18 [11] in $\mathrm{CD}$, as well as the expression of the IL-12(p40) subunit $[5,10,12]$. Our aim was to provide homogeneous information from these cytokines during the period of long-standing inflammation in $\mathrm{CD}$, particularly concerning conflicting aspects found in previous reports, such as the role of IL-18 in the absence of IL-12, or the tissue localization of IL-15. We have studied biopsies from patients with untreated $\mathrm{CD}, \mathrm{CD}$ patients on a gluten-free diet (GFD), and healthy controls. A group of patients with non-CD intestinal inflammation and mild histological abnormalities has been also included, in order to investigate whether the mechanisms involved in CD are exclusive of this pathology or not. Besides cytokine expression in non-stimulated biopsies, we have also analysed the production of IL-15 and IL-18 in a number of biopsies from CD patients on a GFD after in vitro stimulation with gluten. 


\section{Materials and methods}

\section{Study subjects}

Intestinal biopsy specimens were collected from $46 \mathrm{CD}$ patients, 20 adults (median age 28.4 years, range $17-52$ years) and 26 children (median age 4.5 years, range 15 months- 12 years). From these, 37 were untreated (13 adults, 24 children) and nine (seven adults and two children) were on a GFD for at least 3 months. Patients attended the Adult and Paediatric Gastroenterology Clinics, from the Hospital Clínico Universitario of Valladolid, as part of the routine diagnostic procedures for suspicion and/or follow-up of CD. Small bowel biopsies were obtained during upper gastrointestinal endoscopy using a fibergastroscope with forceps in adults (Olympus, Tokyo, Japan), and using a paediatric Crosby capsule in children. Patients with active $\mathrm{CD}$ had an altered biopsy histology, positive antitransglutaminase antibodies and the HLA-DQ2 genotype, and the diagnosis was confirmed by the remission of symptoms and recovery of the histological and serological markers after GFD. Mucosal abnormalities in biopsies were described according to the modified Marsh' classification [13].

The study included two groups of controls. (i) A group of 19 patients with normal biopsy histology, 14 adults (mean age $40 \cdot 7$ years, range $16-53$ years), and five children (mean age 4.37 years, range 18 months to 7 years) who underwent diagnostic investigations due to clinical suspicion of a gastrointestinal disorder that was later ruled out. (ii) A group of 11 adult patients (median age $39 \cdot 6$ years, range $14-80$ years) with non-specific small bowel inflammation, i.e. patients investigated because of clinical suspicion of small bowel disease but having negative serological markers of $\mathrm{CD}$ and mild histological changes in the biopsy, consisting of a nonspecific lamina propria infiltrate, but no villous atrophy.

Informed consent was obtained from patients and/or their parents, and the study protocol was approved by the Ethics Committee of the University Hospital and Faculty of Medicine of Valladolid.

\section{In vitro gluten stimulation of biopsy specimens}

Intestinal biopsies from three CD patients on a GFD were cultured in vitro as previously described [14]. Briefly, biopsies were collected in ice-chilled tissue culture medium containing RPMI 1640 (Cambrex Iberia Products, Barcelona, Spain) supplemented with $10 \%$ heat-inactivated FBS, penicillin $(100 \mathrm{U} / \mathrm{ml})$, streptomycin $(100 \mu \mathrm{g} / \mathrm{ml})$ and fungizone $(0.25 \mu \mathrm{g} / \mathrm{ml})$ (Cambrex). From each patient, one biopsy explant was cultured in medium containing $0.2 \mathrm{mg} / \mathrm{ml}$ of gliadin (Sigma-Aldrich, St Louis, MO, USA), and a second one in culture medium alone. Tissue culture was carried out by immersion in culture dishes placed in a cell incubator with $5 \% \mathrm{CO}_{2}$ at $37^{\circ} \mathrm{C}$. After $3 \mathrm{~h}$ of stimulation, biopsies were washed up with PBS containing $0 \cdot 1 \%$ gentamicine (Cambrex), and cultured again for $21 \mathrm{~h}$ in culture medium. Finally, specimens were collected in RNA-Later (Ambion Inc, Austin, Texas, USA) and snap-frozen until further processing. Proteins from these samples were extracted to perform Western-blot analysis for IL-15 and IL-18, as detailed below.

\section{Sample preparation}

After collection, tissue samples were immediately submerged in $1 \mathrm{ml}$ of RNA-Later ${ }^{\circledR}$ solution (Ambion) and stored at $-20^{\circ} \mathrm{C}$ to preserve RNA integrity. Both total RNA and protein were isolated from each biopsy using the TRIZOL ${ }^{\circledR}$ reagent according to the protocol provided by the manufacturer.

Reverse transcription was carried out by using the SuperScript ${ }^{\circledR}$ First-Strand Synthesis System for reverse transcriptase (RT)-PCR Kit (Invitrogen, Life Technologies, Carlsbad, CA, USA) according to the manufacturer's instructions, using OligodT primers.

\section{Quantitative polymerase chain reaction}

mRNA levels of TNF $\alpha$, IL-12(p35), IL-12(p40), IL-15, IL-18 and IL-23(p19) were determined by real-time PCR using a LightCycler ${ }^{\circledR}$ instrument (Roche Applied Science, Mannheim, Germany). Reactions were performed in a volume of $20 \mu \mathrm{l}$ using the FastStart SYBR Green I MasterMix (Roche Applied Science) and $1 \mu \mathrm{l}$ of thermolabile Uracil DNA Glicosilase (UDG) (Roche Applied Science) to prevent carryover contamination. Cytokine primer sets are described in Table 1. Samples were analysed in triplicate and results, obtained as a ratio of cytokine/ $\beta$-actin mRNA levels, were expressed as arbitrary units.

A standard curve for each gene was made using serial dilutions from the RNA extracted from cell lines, either Jurkatt $\left(6 \times 10^{6}\right.$ cells $)$ or Thp1 $\left(10 \times 10^{6}\right.$ cells $)$, previously stimulated with $10 \mathrm{nM}$ of PMA and $3 \mu \mathrm{M}$ of Ionomycin for $5 \mathrm{~h}$.

\section{Immunohistochemistry}

Cytokine mRNA expression was confirmed by immunohistochemistry in paraffin-embedded tissue specimens from $10 \mathrm{CD}$ patients, and tissue sections from patients with active Crohn's disease were used as positive controls. IL-15 was analysed using two distinct primary antibodies in separate experiments, and IL-18 was detected with a single monoclonal antibody. For antigen unmasking, paraffin sections were boiled in a pressure cooker for $2 \mathrm{~min}$ using either TRIS $10 \mathrm{mM} / \mathrm{EDTA} 1 \mathrm{mM} \mathrm{pH} 8$ (for both antibodies against IL-15) or TRIS buffer $10 \mathrm{mM}$ pH 10 (for IL-18). Endogenous peroxidase was blocked by treatment with $1 \% \mathrm{H}_{2} \mathrm{O}_{2}$ in methanol, and unspecific reactions by using rabbit serum at a dilution of $1 / 50$. Sections were incubated overnight at $4{ }^{\circ} \mathrm{C}$ 
Table 1. PCR conditions.

\begin{tabular}{|c|c|c|c|c|c|}
\hline Cytokine & $\begin{array}{l}\text { Standard } \\
\text { RNA }\end{array}$ & Primers sequence & bp & Annealing & Primers source \\
\hline$\beta$-actin & JK & $\begin{array}{l}\text { FW: } 5^{\prime} \text {-atg ggt cag aag gat tcc tat gtg- } 3^{\prime} \\
\text { RV: } 5^{\prime} \text {-ctt cat gag gta gtc agt cag gtc- } 3^{\prime}\end{array}$ & 359 & 60 & $\begin{array}{l}\text { M. Bongers, E. Liehl, J. Barsig, } \\
\text { Focus 21, } 66 \text { (2006) }\end{array}$ \\
\hline TNF $\alpha$ & Thp1 & $\begin{array}{l}\text { FW: } 5^{\prime} \text {-tca gat cat ctt ctc gaa } \mathrm{cc}-3^{\prime} \\
\text { RV: } 5^{\prime} \text {-cag ata gat ggg ctc ata } c c-3^{\prime}\end{array}$ & 361 & 60 & G. J. Atkins et al. Bone 26, 653 (2000) \\
\hline IL-12(p35) & Thp1 & $\begin{array}{l}\text { FW: } 5^{\prime} \text {-tgt cac cga gaa gct gat gt- } 3^{\prime} \\
\text { RV: } 5^{\prime} \text {-gag gtt tct ggc caa act ga- } 3^{\prime}\end{array}$ & 278 & 68 & Designed with the Primer3 software \\
\hline IL-12(p40) & From manufacturer & From manufacturer & - & - & SearchLC, Heidelberg, Germany \\
\hline IL-15 & JK & $\begin{array}{l}\text { FW: } 5^{\prime} \text {-tgt ctt cat ttt ggg ctg ttt c.- } 3^{\prime} \\
\text { RV: } 5^{\prime} \text {-tcc tcc agt tcc tca cat tct ttg }\end{array}$ & 327 & 60 & $\begin{array}{l}\text { C. Kebelmann-Betzing et al.. } \\
\text { Cytokine 13, } 39 \text { (2001) }\end{array}$ \\
\hline IL-18 & Thp1 & $\begin{array}{l}\text { FW: } 5^{\prime} \text {-gcttga atc taa att atc agt c- } 3^{\prime} \\
\text { RV: } 5^{\prime} \text {-caa att gca tct tat tat cat g- } 3^{\prime}\end{array}$ & 335 & 55 & $\begin{array}{l}\text { T. Tomita et al. J. Infect. Dis. } \\
\text { 183, } 620 \text { (2001) }\end{array}$ \\
\hline IL-23 (p19) & $\mathrm{Jk}$ & $\begin{array}{l}\text { FW: } 5^{\prime} \text {-agc agc tca agg atg gca ctc ag- } 3^{\prime} \\
\text { RV: } 5^{\prime} \text {-cce caa att tcc ctt ccc atc ta- } 3^{\prime}\end{array}$ & 206 & 60 & $\begin{array}{l}\text { A. Wesa, A. Galy. BMC. Immunol } \\
\text { 3, } 14 \text { (2002) }\end{array}$ \\
\hline
\end{tabular}

Cytokine primer sets, PCR products (bp, base pairs), temperature of annealing, and source of the primers used for quantitative PCR.

with a primary antibody to human IL-15 either polyclonal (goat polyclonal SC-1296, Santa Cruz Biotechnology, Santa Cruz, CA, USA), or monoclonal (mouse monoclonal MAB247, R \& D, Minneapolis, MN, USA), and human IL-18 (mouse monoclonal SC-13602, Santa Cruz Biotechnology), at a final dilution of $1 / 50$. Then, sections were treated by the Avidin-Biotin peroxidase complex technique (DakoCytomation, Glostrup, Denmark), using a secondary biotin-labelled IgG antibody, either anti-mouse or anti-goat (DakoCytomation) at a final dilution of $1 / 500$, followed by treatment with Diaminobenzidine (DAB, Bio-Rad Laboratories, Hercules, CA, USA). Harris Hematoxilin was used for counterstaining (Merck, Darmstadt, Germany). As isotype controls, tissue sections were incubated with the corresponding isotype and the usual working concentration of an irrelevant monoclonal antibody.

\section{Western blot analysis}

Total protein was extracted by the TRIZOL method, as mentioned before. In all cases, $10 \mu \mathrm{g}$ of protein were added per well, and separated by using a 15\% acrilamide/bisacrilamide (37.5:1) gel in a mini-Protean II (Bio-Rad Laboratories), and transferred onto PVDF membranes of 0.45 Micron (Pierce Biotechnology Inc., Rockford, IL, USA). Membranes were incubated with primary specific antibodies to human IL-15 (mouse monoclonal MAB247, R \& D) and IL18 (mouse monoclonal SC-13602, Santa Cruz Biotechnology) at a final dilution of $1 / 400$ and $1 / 200$, respectively; a second incubation was carried out with antibodies to mouse IgG labelled with horseradish peroxidase (Amersham Biosciences Europe, Freiburg, Germany), using the chemiluminiscent substrate Lumigen PS-3 (Amersham) and the autoradiography film Hyperfilm ECL (Amersham). Finally, the QuantityOne software (Bio-Rad) was used for band, molecular weight and densitometric analysis. Recombinant human IL-15 (Peprotech, London, UK) and IL-18 (Chemicon, Temecula, CA, USA) were used as positive controls.

\section{Statistical analysis}

Results are expressed as the median value and the interquartile range. Statistical differences in cytokine mRNA expression levels between groups were analysed using the Mann-Whitney $U$-test, and analysis of non-parametric correlation was performed by using the Spearman's rank correlation test. A value of $P<0.05$ was considered statistically significant. The statistical analysis was performed by using the software program SPSS 11.0.

\section{Results}

\section{No differences in cytokine mRNA levels among study groups}

Similar mRNA levels of TNF $\alpha$ were found in untreated CD patients (0.754 [4.559]), CD patients on GFD (0.997 [14.530]), and healthy controls $(0 \cdot 628$ [2.085]). In non-CD inflammatory controls, TNF $\alpha$ mRNA expression $(2 \cdot 700$ [4.208]) is increased above the levels of healthy controls, although differences are not statistically significant, possibly due to the small number of samples (Table 2).

mRNA expression of both IL-12 subunits (p35 and p40) remains below detectable levels in most samples from all study groups. However, p40 is sporadically expressed (proportion of samples with detectable levels in study groups is shown in Table 2). Samples expressing IL-12(p40) mRNA coexpressed also IL-12(p35) mRNA in a high proportion (75\%, six out of eight samples). Nearly all samples show mRNA of IL-23(p19), but its levels do not change in active 
Table 2. Cytokine mRNA expression in different study groups.

\begin{tabular}{|c|c|c|c|c|c|c|}
\hline & $\mathrm{TNF} \alpha^{*}$ & IL-15* & IL-18* & IL-23 (p19)* & IL-12 (p35) & IL-12 (p40)** \\
\hline Normal controls $(n=19)$ & $0.628(2 \cdot 085)$ & $0 \cdot 159(0.329)$ & $8.638(64.532)$ & $0 \cdot 878(1 \cdot 231)$ & $42 \%(8 / 19)$ & $11 \%(2 / 19)$ \\
\hline Untreated CD $(n=36)$ & $0 \cdot 754(4 \cdot 559)$ & $0 \cdot 097(0 \cdot 312)$ & $11 \cdot 084(27 \cdot 263)$ & $0 \cdot 480(3 \cdot 164)$ & $47 \%(17 / 36)$ & $8 \%(3 / 36)$ \\
\hline Treated CD $(n=9)$ & $0.997(14.530)$ & $0.411(1.988)$ & $10 \cdot 278(40 \cdot 474)$ & $0 \cdot 518(3 \cdot 424)$ & $33 \%(3 / 9)$ & $22 \%(2 / 9)$ \\
\hline Inflammation controls $(n=11)$ & $2 \cdot 700(4 \cdot 208)$ & $0 \cdot 161(6 \cdot 813)$ & $20 \cdot 246(39 \cdot 914)$ & $1 \cdot 200(4 \cdot 407)$ & $45 \%(5 / 11)$ & $9 \%(1 / 11)$ \\
\hline
\end{tabular}

${ }^{\star}$ Results expressed as median and interquartile range in arbitrary units. ${ }^{\star \star}$ Results expressed as percentage of samples with detectable levels of mRNA, number of positive samples and total samples.

CD $(0 \cdot 480[3 \cdot 164])$, CD patients on a GFD $(0 \cdot 518[3 \cdot 164])$, non-CD inflammatory controls $(1 \cdot 200$ [4.407]) and healthy controls $(0 \cdot 878[1 \cdot 231])$.

Detectable levels of IL-15 and IL-18 mRNA have been found in nearly all samples, although no significant differences have been observed among patient groups (Table 2).

Children and adult patients with CD show similar mRNA levels for all the cytokines studied.

\section{IL-15 protein is almost absent in long-standing untreated $C D$ patients}

Western-blot analysis of IL-15 from biopsy protein extract (Fig. 1a) shows a low proportion of samples with detectable IL-15 levels, which does not change in active CD (one out of 10 samples), CD patients on a GFD (one out of four samples), non-CD inflammatory controls (one out of eight samples), and healthy controls (one out of twelve samples). Human recombinant IL-15 was used as positive control, showing a positive band of $12.9 \mathrm{kDa}$.

Using the protein extract from gluten-stimulated biopsy explants from CD patients on a GFD, IL-15 was detected in both stimulated and control explants from case I, IL-15 was not detected in case II, and finally, case III showed no IL-15 expression in the control biopsy, while the expression of this cytokine was prominent in the explant stimulated with gliadin (Fig. 1c).

Immunohistochemical analysis of tissue sections from untreated CD patients shows that IL-15 is almost absent (goat polyclonal anti-human-IL-15 antibody) (Fig. 2a). Positive IL-15 expression was found in one out of 10 samples, in which sporadic staining of lamina propria mononuclear cells was detected, but not epithelial expression. In order to ensure sensitivity and specificity of the labelling conditions, tissue slides from active CD patients were stained with a different antibody (mouse monoclonal anti-human-IL-15 antibody), and both the pattern of expression of IL-15 and the proportion of positive samples were similar to previous results (Fig. 2b). We have also analysed tissue sections from Crohn's disease patients, and abundant expression of IL-15 was found in cells from both epithelium and lamina propria (Fig. 2c).

\section{Untreated CD show IL-18 protein expression localized within the crypts}

Western-blot analysis shows that the inactive form of IL-18 $(24 \mathrm{kDa})$ is present in all samples $(n=10)$ from active CD patients, while the active form $(18 \mathrm{kDa})$ is found in eight out of 10 samples (Fig. 1b). In the remaining groups, both forms of IL-18 are sporadically detected: CD patients on a GFD (one out of four samples), non-CD inflammatory controls (two out of eight samples) and healthy controls (three out of 10 samples). By using human recombinant IL-18 as a positive control, a band of $18 \mathrm{kDa}$ corresponding to the active form was detected. Densitometric analysis of the band intensity showed that the active form of IL-18 is increased in active CD patients above that in healthy controls $(P=0.023)$

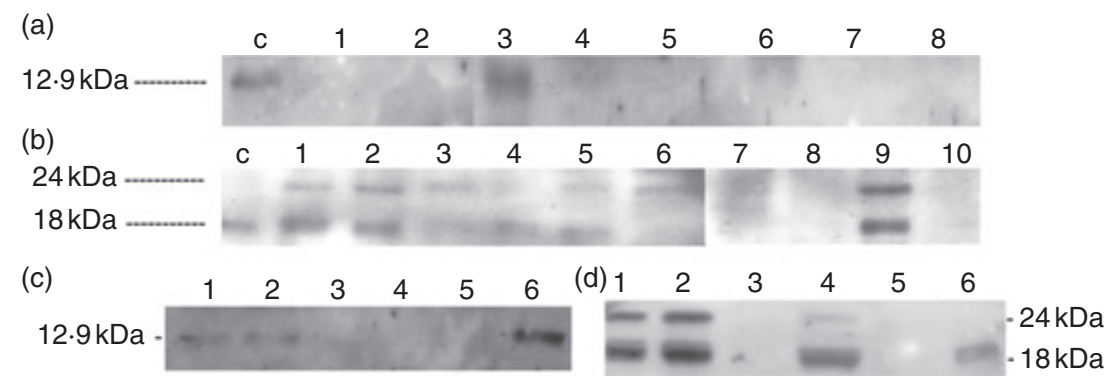

Fig. 1. Western-blot analysis, representative of several experiments. IL-15 (A): human recombinant IL-15, lane 'c'; untreated CD patients, children, lanes 1-2, and adults, lanes 3-4; inflammatory controls, lanes 5-6; healthy controls, lanes 7-8. IL-18 (B): human recombinant IL-18, lane 'c'; untreated CD patients, children, lanes 1-3, and adults, lanes 4-6; inflammatory controls, lanes 7-8; healthy controls, lanes 9-10. IL-15 (C): gliadin stimulated biopsies from three CD patients on a gluten-free diet, culture medium alone, lanes 1, 3 and 5; culture medium supplemented with gliadin $0.2 \mathrm{mg} / \mathrm{ml}$, lanes 2, 4 and 6 . IL-18 (D): gliadin stimulated biopsies from three CD patients on a gluten-free diet, culture medium alone, lanes 1,3 and 5; culture medium supplemented with gliadin $0 \cdot 2 \mathrm{mg} / \mathrm{ml}$, lanes 2,4 and 6 . 

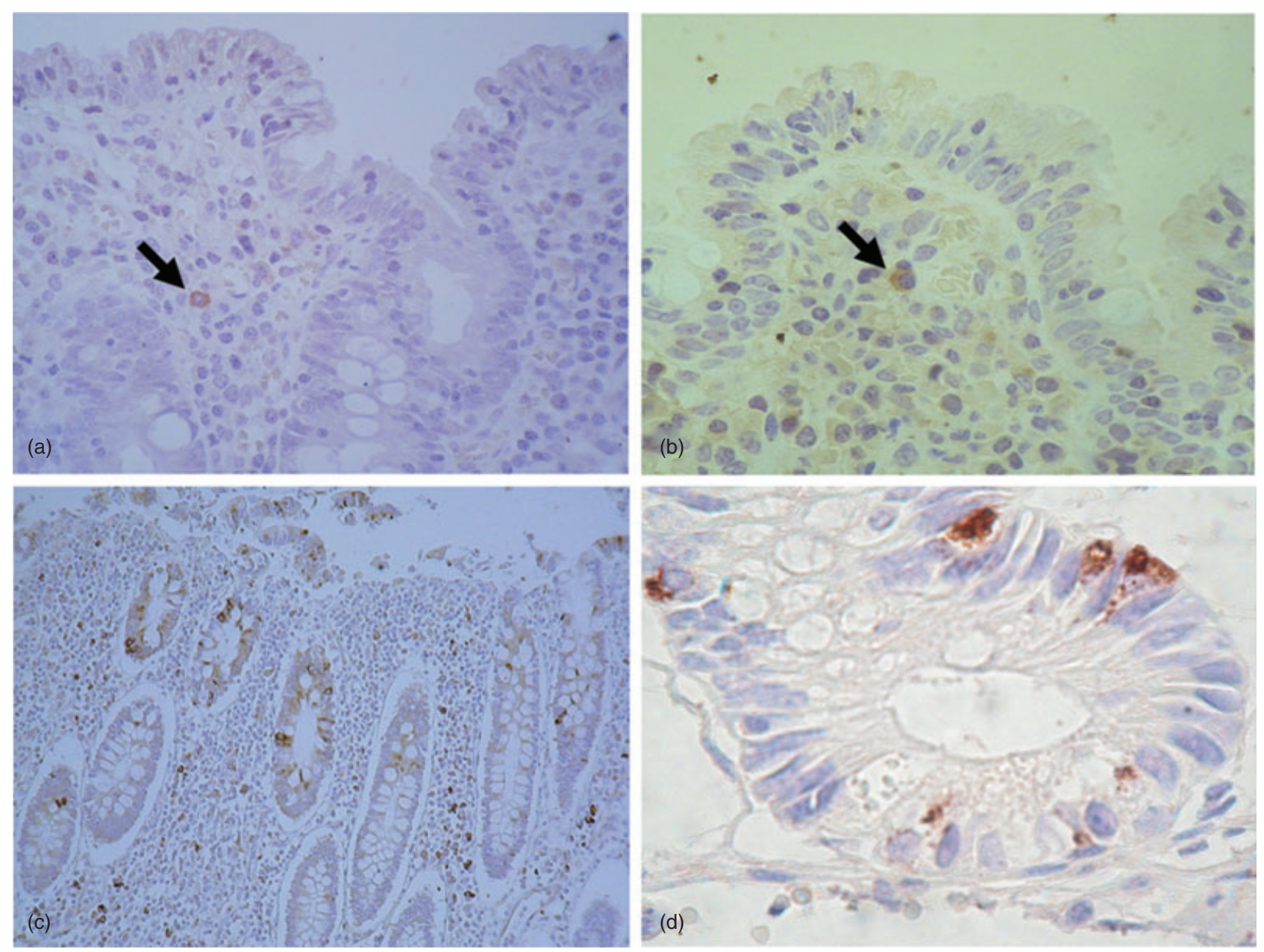

Fig. 2. Immunohistochemistry in paraffin-embedded tissue sections. (a) IL-15 is found sporadically in lamina propria mononuclear cells, but not within the epithelium, CD patient, Goat polyclonal anti-human-IL-15 antibody (400× magnification). (b) IL-15 is found sporadically in lamina propria mononuclear cells, but not within the epithelium, CD patient, mouse monoclonal anti-human-IL-15 antibody (400× magnification). (c) IL-15 expression in tissue section from a Crohn's disease patient, goat polyclonal anti-human-IL-15 antibody (100× magnification). (d) IL-18 is expressed within the crypts, CD patient (400× magnification).

and CD patients on a GFD $(P=0 \cdot 024)$, and, finally, differences with non-CD inflammatory controls did not reach statistical significance (Fig. 3).

Using the protein extracts from gluten-stimulated biopsy explants from CD patients on a GFD, both the active and inactive forms of IL-18 were detected in stimulated and control explants from case I, while in case II both forms were detected only in the gliadin-stimulated explant, and case III showed only the active form of IL-18 in the gliadin-stimulated explant but not in the control explant (Fig. 1d).

With regard to the localization and cellular source of IL-18 (Fig. 2d), immunohistochemical staining showed that IL-18 is expressed within the crypts in active CD biopsies, but not at the surface of the villi, and no positive cells were detected in the lamina propria. The cellular type of the IL-18 producing cells was not identified.

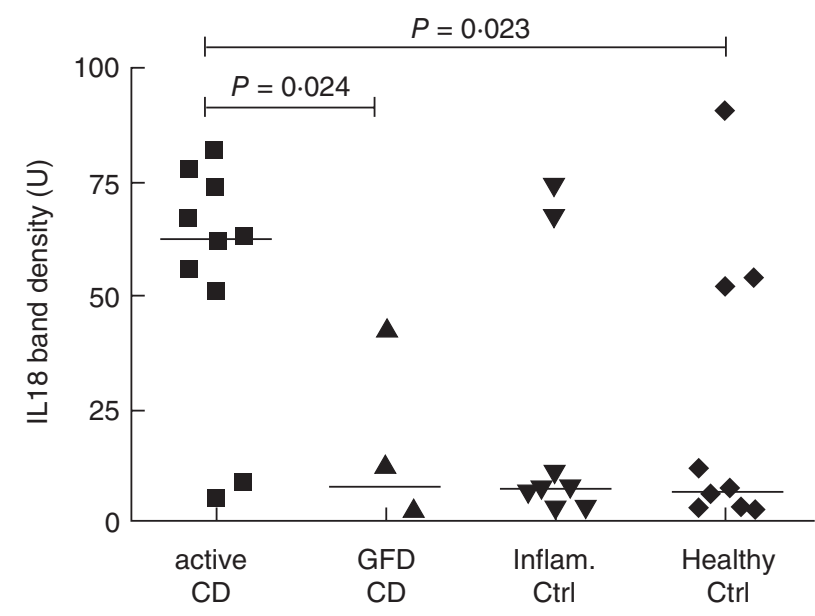

Fig. 3. Densitometric analysis of IL-18 (18 kDa) bands obtained by Western-blot, showing an increased expression in active CD compared with controls and CD patients on a GFD. 


\section{Discussion}

IFN $\gamma$, TNF $\alpha$ or IL-15 play an important role in the initial response to gluten in the intestinal mucosa of CD patients, as has been shown in experimental models using biopsies stimulated with gliadin peptides in vitro $[5,10]$. We have shown that gliadin stimulation of biopsies from CD patients on a GFD may induce the expression at the protein level of both IL-15 and also the active form of IL-18. The role of IL-15 in the early response to gluten, especially as a mediator of epithelial damage, has been previously established [9]. IL-18 has a crucial role in maintaining Th1 responses in human diseases and experimental models, and induces IFN $\gamma$ gene expression and protein secretion alone, or in combination with IL-12 [15,16], although there is little information available about the direct effect of this cytokine on the intestinal mucosa. IL-18 might have a paradoxical effect, inducing apoptosis by enhancing the expression of Fas-ligand [17], while exerting a protective effect on the epithelium by an IL-11-mediated mechanism [18]. However, studies on the expression of IL-18 receptor and other functional studies should be carried out to confirm this hypothesis.

The study of non-stimulated biopsies from patients with active CD reflects that levels of IFN $\gamma[6,7]$ are increased between the episodes of gluten intake, and confirms the presence of a long-standing inflammatory activity beyond the contact with gluten peptides. TNF $\alpha$ mRNA levels increase in biopsies from CD patients after in vitro gluten challenge [5], but this does not occur in non-stimulated biopsies from active $\mathrm{CD}$, as our results, and others [8], have shown. Although IL-12 is the main inducer of IFN $\gamma$ in other intestinal inflammations [19], IL-12 mRNA is expressed in a low number of samples in both untreated CD patients and healthy controls, whereas IL-12(p35) is more frequently expressed, but does not seem to be increased in untreated CD. IL-23 is closely related to IL-12, and both cytokines share the IL-12(p40) subunit [20], although its levels do not increase in active inflammation, as compared with healthy controls. Therefore, neither TNF $\alpha$, nor IL-12, nor IL-23 contributes to the maintenance of the long-standing intestinal inflammation in CD $[5,10,12]$.

Our study, and also a previous report [11], have found protein expression of both the active and inactive forms of IL-18 in nearly all untreated biopsy samples from CD patients. In healthy controls, active IL-18 is only sporadically found, which may correspond to a certain degree of immunological activation observed in some individuals, either CD patients on a GFD, or inflammatory controls. In untreated CD, mRNA levels of IL-18 are similar to those found in healthy controls. This cytokine is mainly regulated at the post-transcriptional level, making possible the finding of the mature form of IL-18 without changes in mRNA levels [11]. We have also found that in active CD, IL-18 is expressed within the epithelial crypts, but it is absent from the lamina propria. Therefore, IL-18 may maintain IFN $\gamma$ expression during the long-standing inflammation time after gluten intake, and its presence within the epithelium suggests a role in enterocyte damage.

No relevant information about IL-15 expression has been found at the mRNA level in our results or in previous studies $[9,10]$. At the protein level, our results by Western-blot and immunohistochemistry show that IL-15 is nearly absent in non-stimulated biopsies, and it is only sporadically found in the lamina propria in a small proportion of samples. Previous studies in non-stimulated biopsies from active CD patients reported abundant positive IL-15 staining within the lamina propria alone [9], and also in the epithelium [10]. Differences between studies might be due to technical issues leading to immunostaining protocols with different sensibility and specificity, and also to differences in the timing from the gliadin intake and biopsy collection.

The third stage of the disease occurs after histological recovery of the mucosa on a GFD, where neither IFN $\gamma$ levels nor the remaining cytokines studied are increased, as compared with healthy controls. These changes suggest that the inflammatory activity disappears in CD patients on a GFD.

We have also studied patients with non-CD inflammatory conditions and mild histological alterations in the intestine. However, contrary to CD, no changes were found in IL-18 levels, or in the remaining cytokines analysed. Therefore, at the time of sample collection, there is no increase in cytokine levels above those of healthy controls, and the mucosal damage may have been induced by past episodes of inflammation.

In conclusion, peaks of cytokine expression may be directly associated with the episodes of gluten intake in active $\mathrm{CD}$, in which an early response with high IFN $\gamma$ levels is driven by IL-18 and probably IL-15, alternating with periods of long-standing inflammation characterized by moderate IFN $\gamma$ levels and maintained by IL-18 alone.

\section{Acknowledgements}

This work was supported by grants from the Instituto de Salud Carlos III, Spanish Ministry of Health (PI020895, 02/3068), Junta de Castilla y León (VA057/04, and SAN1052VA02/05), a fellowship from Junta de Castilla y León (O.C. 14/11/03-O.R.26/01/0), and Sweden Diagnostics affiliated to Pharmacia Diagnostics.

\section{References}

1 Strober W, Kelsall B, Fuss I et al. Reciprocal IFN-gamma and TGFbeta responses regulate the occurrence of mucosal inflammation. Immunol Today 1997; 18 (2):61-4.

2 León AJ, Garrote JA, Arranz E. Citocinas en la patogenia de la enfermedad celiaca. Med Clin (Barc) 2005; 125:508-16.

3 MacDonald TT. Effector and regulatory lymphoid cells and cytokines in mucosal sites. Curr Top Microbiol Immunol 1999; 236:11335. 
4 Maiuri L, Picarelli A, Boirivant M et al. Definition of the initial immunologic modifications upon in vitro gliadin challenge in the small intestine of celiac patients. Gastroenterology 1996; 110 (5):1368-78.

5 Nilsen EM, Jahnsen FL, Lundin KE et al. Gluten induces an intestinal cytokine response strongly dominated by interferon gamma in patients with celiac disease. Gastroenterology 1998; 115 (3):55163.

6 Salvati VM, Bajaj-Elliott M, Poulsom R et al. Keratinocyte growth factor and coeliac disease. Gut 2001; 49 (2):176-81.

7 Westerholm-Ormio M, Garioch J, Ketola I, Savilahti E. Inflammatory cytokines in small intestinal mucosa of patients with potential coeliac disease. Clin Exp Immunol 2002; 128 (1):94101.

8 Forsberg G, Hernell O, Melgar S, Israelsson A, Hammarstrom S, Hammarstrom ML. Paradoxical coexpression of proinflammatory and down-regulatory cytokines in intestinal $\mathrm{T}$ cells in childhood celiac disease. Gastroenterology 2002; 123 (3):667-78.

9 Maiuri L, Ciacci C, Auricchio S, Brown V, Quaratino S, Londei M. Interleukin 15 mediates epithelial changes in celiac disease. Gastroenterology 2000; 119 (4):996-1006.

10 Mention JJ, Ben Ahmed M, Begue B et al. Interleukin 15: a key to disrupted intraepithelial lymphocyte homeostasis and lymphomagenesis in celiac disease. Gastroenterology 2003; 125 (3):730-45

11 Salvati VM, MacDonald TT, Bajaj-Elliott M et al. Interleukin 18 and associated markers of $\mathrm{T}$ helper cell type 1 activity in coeliac disease. Gut 2002; 50 (2):186-90.

12 Monteleone G, Pender SL, Alstead E et al. Role of interferon alpha in promoting $\mathrm{T}$ helper cell type 1 responses in the small intestine in coeliac disease. Gut 2001; 48 (3):425-9.

13 Working Group of the United European Gastroenterology Week. When is a coeliac a coeliac? Report of a working group of the United European Gastroenterology Week in Amsterdam. Eur J Gastroenterol Hepatol 2001; 13:1123-8.

14 Fais S, Maiuri L, Pallone F et al. Gliadin induced changes in the expression of MHC-class II antigens by human small intestinal epithelium. Organ culture studies with coeliac disease mucosa. Gut 1992; 33 (4):472-5.

15 Pizarro TT, Michie MH, Bentz M et al. IL-18, a novel immunoregulatory cytokine, is up-regulated in Crohn's disease: expression and localization in intestinal mucosal cells. J Immunol 1999; 162 (11):6829-35.

16 Robinson D, Shibuya K, Mui A et al. IGIF does not drive Th1 development but synergizes with IL-12 for interferon-gamma production and activates IRAK and NFkappaB. Immunity 1997; 7 (4):571-81.

17 Dinarello CA. Interleukin-18. Methods 1999; 19 (1):121-32.

18 Reuter BK, Pizarro TT. Commentary. The role of the IL-18 system and other members of the IL-1R/TLR superfamily in innate mucosal immunity and the pathogenesis of inflammatory bowel disease: friend or foe? Eur J Immunol 2004; 34 (9):2347-55.

19 Monteleone G, Biancone L, Marasco R et al. Interleukin 12 is expressed and actively released by Crohn's disease intestinal lamina propria mononuclear cells. Gastroenterology 1997; 112 (4):1169_ 78.

20 Hunter CA. New IL-12-family members. IL-23 and IL-27, cytokines with divergent functions. Nat Rev Immunol 2005; 5 (7):521-31. 